\title{
Portfolio Analysis of Solar Photovoltaics: Quantifying the Contributions of Locational Marginal Pricing and Power on Revenue Variability
}

\author{
Katrina Kumpf ${ }^{a}$, Seth Blumsack ${ }^{\mathrm{a}}$, George S. Young ${ }^{\mathrm{b}}$, Jeffrey R. S. \\ Brownson ${ }^{\mathrm{a}, *}$ \\ ${ }^{a}$ Department of Energy and Mineral Engineering, College of Earth and Mineral Sciences, \\ Pennsylvania State University, PA 16802, USA \\ ${ }^{b}$ Department of Meteorology, College of Earth and Mineral Sciences, Pennsylvania State \\ University, PA 16802, USA
}

\begin{abstract}
For firms developing and managing portfolios of PV assets in utility, commercial, and residential markets the financial performance of the portfolio is a highly relevant business decision factor. Informative metrics are required to quantify the revenue variance of a spatially distributed portfolio of PV assets as well as the individual asset. Financial analysis uses a risk measure known as 'beta' to describe the movement of assets relative to a broader portfolio. We define a measure termed the 'solar beta' that describes the movement of solar PV revenues at a given site with that of a portfolio of sites. The solar beta incorporates correlation between a site and portfolio, and the volatility of a site relative to the portfolio. We also derive and discuss a method to decompose revenue variance of individual PV assets into components representing price, power, and the interplay among diurnal/seasonal cycles and prevailing weather conditions. This decomposition and the solar beta are illustrated using nine modeled sites following a N-S trend within the PJM Interconnection. We find that revenue variance of a PV asset depends more on diurnal, seasonal, and meteorological fluctuations than on price fluctuations at a particular site. Specifically, the contribution of power variance exceeds the contribution of price variance by roughly a factor of five. Changes in mean market price have a larger effect on revenue variance compared to
\end{abstract}

\footnotetext{
*Corresponding author. Tel:+814-867-4227

Email address: solarpower@psu.edu (Jeffrey R. S. Brownson)
} 
a proportional change in mean power production. The solar beta was found to be near 1.0 for most sites, indicating strong covariance within the portfolio due in large part to high correlation rather than similar volatility ratios. Lower beta values were found for sites at the perimeters of the study region, due to change in climate regime and population-power consumption cycles, implying portfolio risk reduction when these sites are included.

Keywords: photovoltaics, locational marginal pricing, portfolio analysis, beta

\section{Introduction}

The amount of PV electricity generation and total capacity has increased dramatically over the past decade. In the USA, PV technologies exhibited a 4000-fold increase in net annual generation over a span of ten years, climbing from $2 \mathrm{GWh}$ in 2003 to 8,300 GWh in 2013.[1] The observed exponential growth trend in net power generation has created opportunities for individual firms to own and manage multiple PV assets. Firms such as SunEdison and SolarCity are developing and managing large portfolios of PV assets for utility, commercial, and residential markets. Likewise, the solar project finance firm Mosaic enables smaller-scale investors to collaborate in the construction of PV asset portfolios. For such firms and other like them, the financial performance of the portfolio, in complement with the individual assets, is thus the relevant business decision factor. As such, they require informative metrics quantifying revenue variance of a spatially distributed portfolio of PV assets.

Applying the tools of portfolio analysis to multisite PV asset assessment provides a model for evaluating the contribution of specific sites to revenue variance over space and time. For individual PV assets, revenue variance depends on the variances of both price and power and their co-fluctuations. The decomposition of revenue variance into price and power variance and cofluctuation terms offers detailed insight into the market and meteorological origins of this risk factor. While such decompositions are routine within fluid dynamics, they are not yet common in energy economics. This approach will be addressed in subsequent sections.

For a portfolio of PV assets, revenue variance depends on the covariance of the individual assets. In financial portfolio management the $\beta$ metric is used as a measure of return variance for a particular asset relative to movements 
in the general market.[2,3] An equivalent formulation may be derived for revenue variance for PV portfolios, which we term the 'solar beta.' This version of beta captures the impact of PV assets progressively added to the portfolio on revenue variance.

These new approaches to financial portfolios in solar are distinct from the works of Hoff and Perez, who showed that a geographically-dispersed portfolio of variable renewables (both solar and wind) can reduce aggregate output power variability.[4, 5] This information is of critical importance to grid operators. In contrast, the new approaches described here quantify the contributors to revenue variance, information of importance to firms owning and managing portfolios of multisite PV assets.

The eastern portion of the PJM Interconnection has been used as a case study. PJM is a Regional Transmission Organization whose footprint covers all or part of 13 states and the District of Columbia. One relevant feature of the PJM market for our analysis is that its market prices are determined with high temporal and spatial granularity. These prices, known as locational marginal prices (LMP, here referred to as "market price"), are published on an hourly basis for thousands of locations within the PJM footprint, indicating the value of providing power to a specific location at a specific time. This research explores the convolved and separate effects of energy production and market price on the overall revenue of photovoltaic arrays in nine different locations throughout the eastern portion of the PJM footprint.

\section{Methodology}

Nine geographically distributed sites within the PJM Interconnection were used for this analysis. The operational zone for each site and their coordinates are shown in Table 1. The sites are also displayed as mapped locations in Figure 1. The common time step (hourly) reflects the finesetgrained time step at which PJM publishes its LMP data. A period of seven years was used, from 2003 to 2009, for which publicly available hourly solar GHI and DNI data corresponded with available LMP data for the chosen PJM zones.

The sites were spaced across the region so as to reflect a variety of solar availability and pricing conditions across the portfolio. Each site was located in a unique operational zone within the PJM footprint, and sites were spaced $>60 \mathrm{~km}$ apart so that the the hourly step for irradiation data would not yield overlap among sites. Spacing was selected to preclude advection of clouds 
from one site to another on the hourly time scale for which our PJM market data is available. Several papers have already noted for cloud motion and speed assessments, that typical wind speeds in the boundary layer tend to be much less than $\sim 60 \mathrm{~km}$ per 1 hour time step, which is why that spacing is used in this study. $[6,7,8]$

Table 1: Selected locations for analysis within the PJM network of the eastern coast, reflecting the corresponding electricity zones and geospatial coordinates.

\begin{tabular}{llc}
\hline Site Name & Corresponding PJM Zone & Site Coordinates \\
\hline PENLC & Pennsylvania Electric Co (PA) & $41.95 \mathrm{~N} 77.45 \mathrm{~W}$ \\
PPL & PPL Electric Utilities (PA) & $41.75 \mathrm{~N} 75.75 \mathrm{~W}$ \\
JCPL & Jersey Central Power Light (NJ) & $41.25 \mathrm{~N} 74.75 \mathrm{~W}$ \\
PSEG & Public Services Electric \& Gas (NJ) & $40.55 \mathrm{~N} 74.65 \mathrm{~W}$ \\
PECO & PECO Energy (PA) & $40.15 \mathrm{~N} 75.35 \mathrm{~W}$ \\
AE & Atlantic Electric Co (NJ) & $39.55 \mathrm{~N} 74.55 \mathrm{~W}$ \\
BGE & Baltimore Gas \& Electric (MD) & $39.35 \mathrm{~N} 76.65 \mathrm{~W}$ \\
PEPCO & Potomac Electric Power Co (MD) & $38.35 \mathrm{~N} 76.65 \mathrm{~W}$ \\
DPL & Delmarva Power \& Light (VA) & $37.35 \mathrm{~N} 75.95 \mathrm{~W}$ \\
\hline
\end{tabular}

The solar irradiation data were acquired from Solar Anywhere®, an online database of solar irradiation. [9] The data sets provided hourly global horizontal irradiance (GHI), direct normal irradiance (DNI), and diffuse horizontal irradiance (DIF), all with units of watts per square meter $\left(\mathrm{W} / \mathrm{m}^{2}\right)$. Only GHI and DNI were needed for the power simulations described below. The hourly irradiance values had a resolution of $10 \mathrm{~km}$ by $10 \mathrm{~km}$.

The LMP data was downloaded directly from the PJM website at an hourly timescale in units of dollars per megawatt-hour (\$/MWh).[10] Energy information was available in real-time as well as day-ahead market. Realtime data was used for this study in order to compare each hourly value to its corresponding solar irradiation value. These data sets were downloaded and then organized by zones for the nine different locations chosen (listed in Table 1).

\subsection{Power Simulations}

The solar irradiation data (GHI and DNI) was used to simulate PV production using the TRaNsient SYstems Simulation (TRNSYS) modeling software.[11] TRNSYS is a robust dynamic simulation tool, allowing data to be input and then modeled based on parameters for solar photovoltaics, solar 


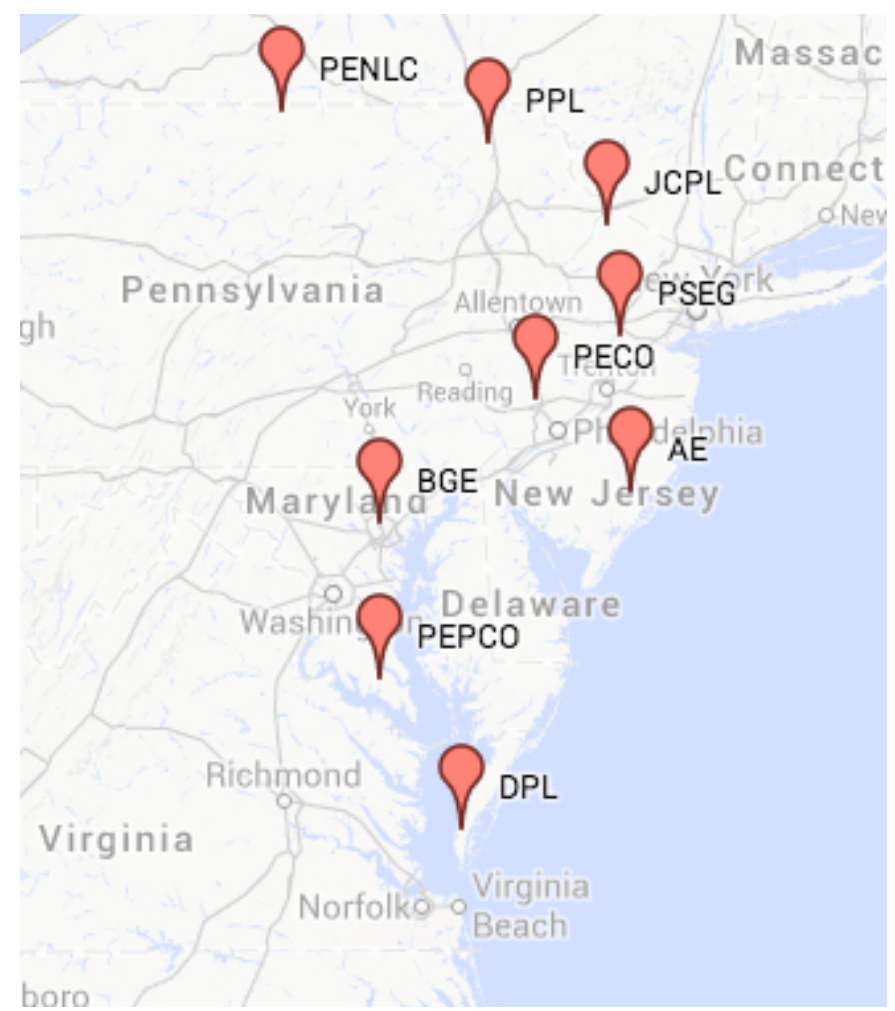

Figure 1: Map of locations for for analysis selected within the PJM Interconnection, within the PJM network of the eastern coast, reflecting the corresponding electricity zones and geospatial coordinates.

thermal, hydropower, and other renewable energies. Figure 2 demonstrates the graphical model components used for this experiment. The processors used have been labeled in the image according to their function. Details for the component specifications within the TRNSYS model can be found in the supplementary material, Appendix A.

A SunPower Corporation $\mathbb{R}$ X20 series $250 \mathrm{~W}$ panel was chosen as the basis for the TRNSYS simulations. All of the necessary parameters and inputs required for the TRNSYS model were taken from the panel's spec sheet available from the manufacturer.[12] At each of the nine sites, an array of 100 panels was simulated to represent a commercial or small-scale utility system of approximately $25 \mathrm{~kW}_{p}$ nameplate capacity. 


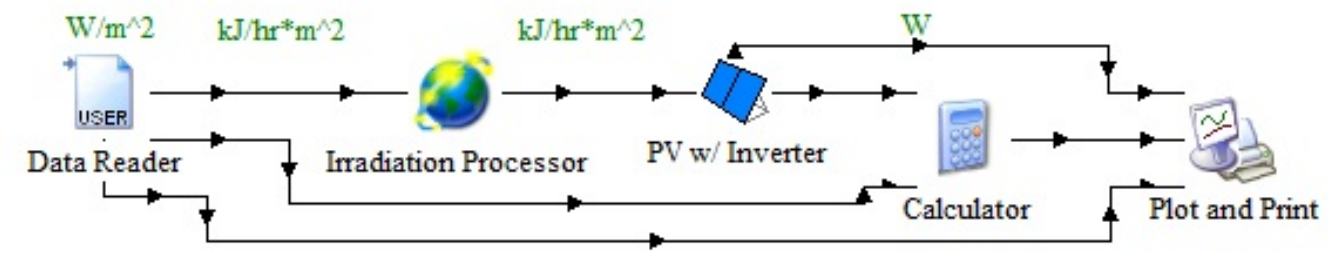

Figure 2: TRNSYS model of PV power production simulations at a single location

\subsection{Individual PV Assets: Revenue Variance}

The derivation and discussion below follow the notation conventions of atmospheric fluid dynamics, the field which inspired this revenue variance decomposition approach.[13] Revenue from a PV asset is the product of two variables: electricity price and power. The variance of revenue is therefore also a function of these two variables. We define $A$ as Revenue $(\$ / \mathrm{h}), B$ as Market Price (\$/MWh), and $C$ as Average Hourly Power (MW). Thus:

$$
A=B * C
$$

captures the relationship between revenue, price and power.

A particular value of a random variable $(X)$ equals the sum of that point's fluctuation from the mean $\left(X^{\prime}\right)$ and the overall mean of the variable $(\bar{X})$. In other words: $B=\bar{B}+B^{\prime}$ and $C=\bar{C}+C^{\prime}$. Using this definition the variance of $A$ can be expressed in terms of the mean and fluctuating components of $B$ and C. Note that by this definition the mean of any fluctuation is zero and mean quantities can be factored out of averages.

Thus, by definition

$$
\operatorname{Var}(A)=\overline{(A-\bar{A})^{2}}=\overline{A^{\prime 2}}
$$

Using the definitions above, the $\overline{(A-\bar{A})^{2}}$ term in Eq. 2 can be decomposed as follows:

$$
\begin{aligned}
\operatorname{Var}(A)= & \bar{B}^{2} \overline{C^{\prime 2}}+\bar{C}^{2} \overline{B^{\prime 2}}+2 \bar{B} \overline{B^{\prime} C^{\prime 2}}+2 \bar{C} \overline{B^{\prime 2} C^{\prime}} \\
& +2 \bar{B} \bar{C} \overline{B^{\prime} C^{\prime}}+\overline{B^{\prime 2} C^{\prime 2}}-\overline{B^{\prime} C^{\prime}}
\end{aligned}
$$

Eq. 3 (derived in Supplemental document, Appendix B) allows the revenue variance to be expressed in terms of the means and co-fluctuations of price and power. 


\subsection{Portfolio Assessment: The Solar Beta}

To measure the contribution of a specific PV site to the revenue variance of a geographically distributed portfolio of PV assets, we adapt the concept of 'beta' from finance theory. Beta is traditionally used as a metric to compare the variance of returns from a single asset (like a stock) with that of a portfolio of assets or the broader asset market. The solar beta differs from financial beta in that it is based on revenue $(\mathrm{A})$, rather than return (since the physical assets in photovoltaic systems are not typically bought and sold in high volumes as would be the case for financial assets), and the solar beta is relative to a portfolio of $p$ assets, rather than the entire market. Given A, the solar beta is defined as:

$$
\beta=\frac{\operatorname{Covariance}\left(A_{p}, A_{i}\right)}{\operatorname{Variance}\left(A_{p}\right)}=\operatorname{Correlation}\left(A_{p}, A_{i}\right) \sqrt{\frac{\operatorname{Variance}\left(A_{i}\right)}{\operatorname{Variance}\left(A_{p}\right)}}
$$

where $A_{p}$ is the portfolio revenue, and $A_{i}$ is the revenue from the $i^{\text {th }} \mathrm{PV}$ asset.

The solar beta is thus the product of two factors: 1) the correlation of $A$ for a single asset with $A$ for a portfolio of PV assets, and 2) the relative volatility (the square root of the ratio of variances) of $A$ at the $i^{\text {th }}$ site compared to that for the portfolio of $\mathrm{PV}$ assets, i.e. the volatility ratio: $\sqrt{\operatorname{Var}\left(A_{i}\right) / \operatorname{Var}\left(A_{p}\right)}$. For example, the revenue from a PV asset with a beta value of +1 fluctuates in perfect synchronization with that from the portfolio, while conversely the revenue from an asset with a negative beta fluctuates in the opposite direction from that of the portfolio. [2, 3]

Since the beta metric is a function of both correlation and volatility ratio, a low solar beta value could have two different causes. Low solar beta could indicate either 1) the revenue from a PV asset has low correlation with that of the portfolio, or 2) the revenue from a PV asset has low variance relative to that of the portfolio. In either case, a PV asset with a low solar beta indicates that the individual asset is a potential "hedge" for the portfolio (i.e., adding the low-beta asset reduces revenue variance for the portfolio). The solar beta is calculated on a site-by-site basis, evaluating the contribution of each individuals site's variability to the variability of the portfolio. 


\section{Results}

\subsection{Individual PV Assets: Revenue Variance}

Table 2 displays the mean values of market price (B) and average power (C), while Table 3 displays the revenue variance and its decomposition for each of the sites, illustrating the contributions of market price and average power, both of which reflect prevailing weather conditions at the sites. The following decomposition terms are described in association with their respective minimum/maximum percent contributions to the total variance among the portfolio of sites (listed in order of appearance in Eq. 3).

Table 2: Mean values for Market Price (B) and Average Hourly Power (C), expressed for each site according to evaluation period of seven years.

\begin{tabular}{llc}
\hline Location & Mean Price $(\$ / M W h)$ & Mean Power $\left(M W \times 10^{-3}\right)$ \\
\hline PENLC & 48.14 & 2.60 \\
PPL & 52.78 & 2.58 \\
JCPL & 55.65 & 2.72 \\
PSEG & 57.21 & 2.78 \\
PECO & 54.18 & 2.78 \\
AE & 56.78 & 2.86 \\
BGE & 57.10 & 2.87 \\
PEPCO & 57.73 & 2.96 \\
DPL & 55.21 & 3.04 \\
\hline
\end{tabular}

Table 3: Values for revenue variance (A) and the terms of its decomposition for each of the sites. Where $\mathrm{B}=$ Market Price, and $\mathrm{C}=$ Average Hourly Power. Note: variance units are defined as the square of the units of the variable, hence $\operatorname{Var}(A)$ is $(\$ / \mathrm{h})^{2}, \operatorname{Var}(B)$ would be $(\$ / \mathrm{MWh})^{2}$, and $\operatorname{Var}(C)(\mathrm{MW})^{2}$.

\begin{tabular}{lcccccccc}
\hline Location & $\operatorname{Var}(A)$ & $\bar{B}^{2} \overline{C^{\prime 2}}$ & $\bar{C}^{2} \overline{B^{\prime 2}}$ & $2 \bar{B} \overline{B^{\prime} C^{\prime 2}}$ & $2 \bar{C} \overline{B^{\prime 2} C^{\prime}}$ & $2 \bar{B} \bar{C} \overline{B^{\prime} C^{\prime}}$ & $\overline{B^{\prime 2} C^{\prime 2}}$ & $-{\overline{B^{\prime} C^{\prime}}}^{2}$ \\
\hline PENLC & 0.082 & 0.043 & 0.006 & 0.010 & 0.002 & 0.005 & 0.017 & -0.000 \\
PPL & 0.109 & 0.050 & 0.009 & 0.014 & 0.004 & 0.006 & 0.026 & -0.001 \\
JCPL & 0.133 & 0.061 & 0.012 & 0.015 & 0.005 & 0.008 & 0.033 & -0.001 \\
PSEG & 0.143 & 0.065 & 0.011 & 0.018 & 0.006 & 0.009 & 0.034 & -0.001 \\
PECO & 0.128 & 0.059 & 0.011 & 0.014 & 0.006 & 0.008 & 0.031 & -0.001 \\
AE & 0.163 & 0.067 & 0.015 & 0.019 & 0.010 & 0.010 & 0.044 & -0.001 \\
BGE & 0.164 & 0.068 & 0.016 & 0.016 & 0.010 & 0.009 & 0.045 & -0.001 \\
PEPCO & 0.184 & 0.072 & 0.018 & 0.019 & 0.012 & 0.011 & 0.053 & -0.001 \\
DPL & 0.147 & 0.068 & 0.014 & 0.016 & 0.005 & 0.009 & 0.035 & -0.001 \\
\hline
\end{tabular}


The first two terms in the decomposition $\left(\bar{B}^{2} \overline{C^{\prime 2}}\right.$ and $\left.\bar{C}^{2} \overline{B^{\prime 2}}\right)$ capture the individual contributions of power variance and price variance to the revenue variance. The remaining five terms capture the joint contribution of power and price fluctuations resulting from their co-fluctuation. The first two terms together explain only $49-59 \%$ of the revenue variance, though they tend to explain a higher proportion of revenue variance for the sites located in zones that frequently experience transmission congestion (and thus higher market price spikes than other zones in the PJM footprint). Even so, quantifying the effect of co-fluctuation between price and power fluctuations is essential to understanding the revenue variability of each individual PV asset.

Inspection shows the individual variance contributions are not of equal strength $\left(\bar{B}^{2} \overline{C^{\prime 2}}: 39-52 \% ; \bar{C}^{2} \overline{B^{\prime 2}}: 7-10 \%\right)$. Algebraic manipulation of Eq. 5 reveals that the ratio of $\bar{B}^{2} \overline{C^{\prime 2}} / \bar{C}^{2} \overline{B^{\prime 2}}$ is equivalent to the square of the ratio of coefficients of price and power. Thus, the relative scale of these two terms results from power having a greater variability relative to price by a factor of 5 (this primarily reflects diurnal variations in solar PV system output).

$$
\frac{\bar{B}^{2} \overline{C^{\prime 2}}}{\bar{C}^{2} \overline{B^{\prime 2}}}=\frac{\bar{C}^{2} / \overline{C^{\prime 2}}}{\bar{B}^{2} / \overline{B^{\prime 2}}}=\left(\frac{\text { coeff. of var. for power }}{\text { coeff. of var. for price }}\right)^{2}
$$

$\bar{B}^{2} \overline{C^{\prime 2}}$ : This term is the square of the mean price times the variance of power, and explains $39-52 \%$ of observed revenue variance. The diurnal cycle observed in this term reflects that of PV power output as seen for the PECO site in Figure 3 (Subfigure B relative to Subfigure D).

$\bar{C}^{2} \overline{B^{\prime 2}}$ : This term is the square of mean power times the variance of price, and explains $7-10 \%$ of observed revenue variance. The diurnal cycle reflected in this term is thus the diurnal price cycle, not the power cycle as seen for the PECO site in Figure 3 (Subfigure A relative to Subfigure C). This term also reflects price variability over longer time scales, where some years experience much higher price spikes than others (not shown). It was found that these high-price events are not confined to any particular season.

Each of the remaining terms reflects the effects of fluctuations in both power and price. Three of them include triple or quadruple products of these fluctuations making their interpretation nontrivial. Each triple product term is the covariance of one fluctuating variable with the square of the other. The 

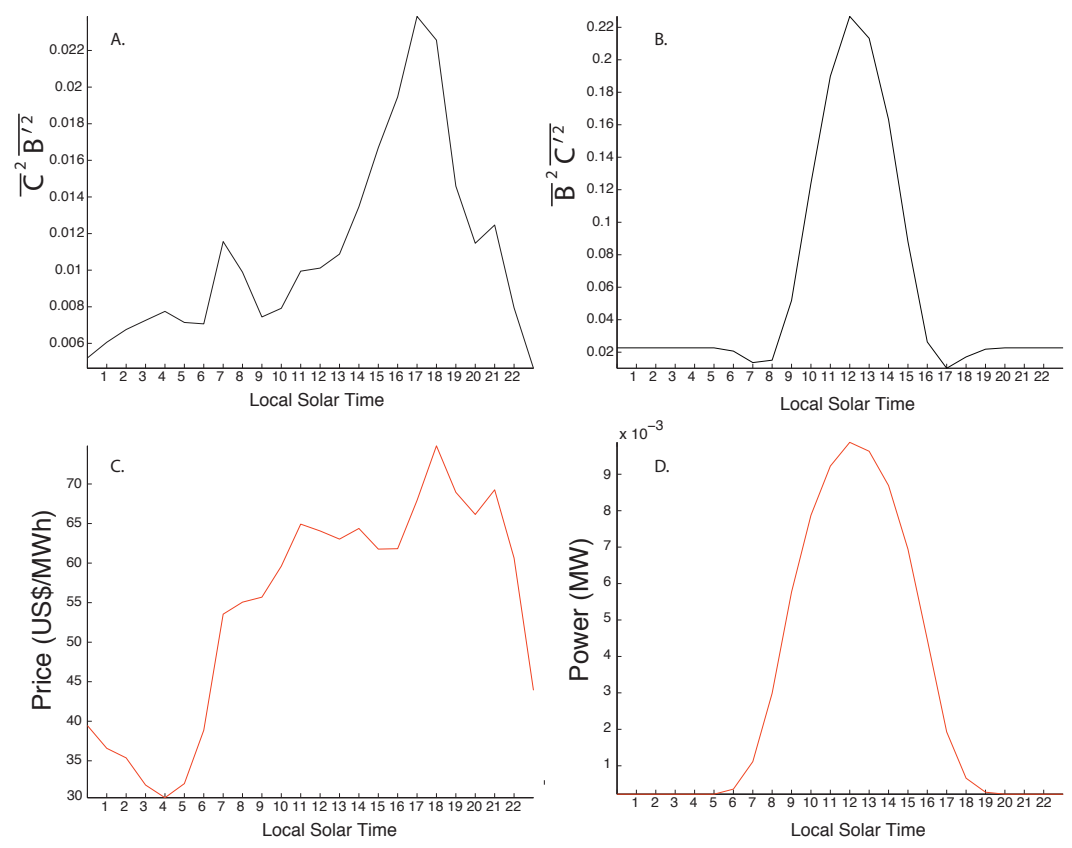

Figure 3: Plots of average diurnal behavior for A) $\bar{C}^{2} \overline{B^{\prime 2}}$ and B) $\bar{B}^{2} \overline{C^{\prime 2}}$, relative to C) Price (B), and D) Power (C), respectively.

quadruple product term is the covariance of the squares of two fluctuating variables. The other two terms are easier to interpret as they include only the covariance of price and power.

$2 \bar{B} \overline{B^{\prime} C^{2}}$ : This triple product term is the mean price times the covariance of power fluctuations with the square of price fluctuations, and explains $10-13 \%$ of observed revenue variance. Thus, the term's diurnal cycle (PECO site, Figure 4) has the same general shape as $\bar{B}^{2} \overline{C^{\prime 2}}$, reflecting the solar diurnal cycle. The inclusion of price fluctuations in the triple product accounts for the elevated values in the evening and the negative values in the predawn hours. A time series plot of this term for the 7 years (not shown) reveals that the positive daytime contribution is frequently larger than the negative nighttime contribution.

$2 \bar{C} \overline{B^{2} C^{\prime}}$ : This triple product term is the mean power times the covariance of the square of price fluctuations with the power fluctuations, and explains $3-9 \%$ of observed revenue variance. Thus, the term's diurnal 


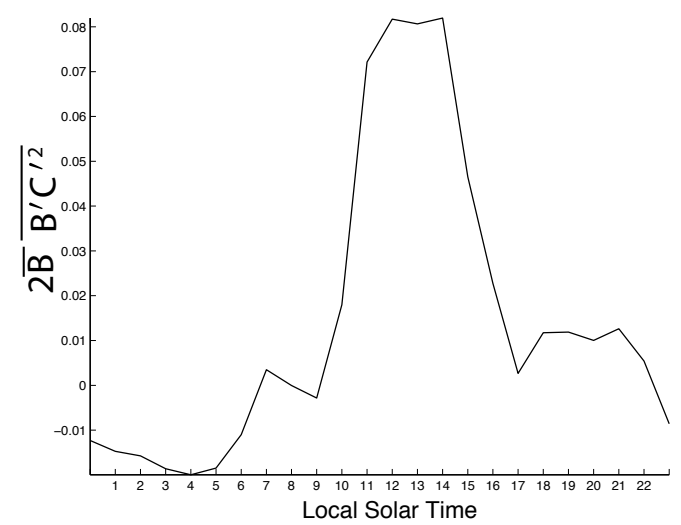

Figure 4: Plot of average diurnal behavior for the mean price times the covariance of power fluctuations with the square of price fluctuations $\left(2 \bar{B} \overline{B^{\prime} C^{\prime 2}}\right)$.

cycle (PECO site, Figure 5 A.) reflects the broad daytime into evening peak in price as modulated by the power fluctuation cycle (about the mean of Figure $5 \mathrm{~B}$, for the PECO site.) which reaches a peak at noon and changes sign around 7 am and $6 \mathrm{pm}$.

$2 \bar{B} \bar{C} \overline{B^{\prime} C^{\prime}}$ : This term is just the mean price times the mean power times the covariance of price and power. It explains $6-7 \%$ of observed revenue variance. Thus, the term reaches a maximum around noon when price and power are both large (not shown). Even multiplied by mean price and power the covariance contribution is small. Thus, including variance term as a simplistic improvement on the sum of the two variance terms is not sufficient to provide a useful guide for decision making for revenue risk management.

$\overline{B^{\prime 2} C^{\prime 2}}$ : This quadruple product term is the covariance of the square of the price fluctuations with the square of the power fluctuations, and explains $21-29 \%$ of observed revenue variations. Thus, the diurnal cycle of this term reflects the midday peak of solar power, but with the maximum delayed by about one hour because of the afternoon increase in price.

$-{\overline{B^{\prime} C^{\prime}}}^{2}$ : This term is the square of the covariance of price and power. It makes a very small contribution to revenue variance of approximately $1 \%$. 


\subsection{Portfolio Assessment: Solar Beta}

The annual values for beta, site correlation, and volatility ratio of revenue are displayed in Figure 6. The results for the the revenue-based solar beta yields values near 1.0 for most of the sites. This occurs because the correlation of most sites with the portfolio is 0.9 while the volatility ratio is slightly above 1.0. Thus, most sites neither add nor subtract from the volatility of the portfolio. Sites at the Northern and Southern ends of the assessed region exhibit lower beta values. Low beta values near the domain ends appear to be influenced more by low correlation with the broader portfolio of sites, although the northernmost and southernmost site also exhibit the lowest volatility ratios relative to the portfolio of sites as a whole. Thus, differences in timing of fluctuations at these sites are a somewhat large contributer to low betas as compared to differences in volatility ratios. [14]

From a meteorological perspective, those stations with beta near 1.0 are all found within the Mid-Atlantic temperate climate regime, and are close enough to each other to be affected by the same weather systems. In contrast, the sites at the N-S perimeter of the portfolio region are influenced by different climate regimes. The northern-most site is under the influence of the Great Lakes, while the southern site experiences subtropical weather during the warm season. 

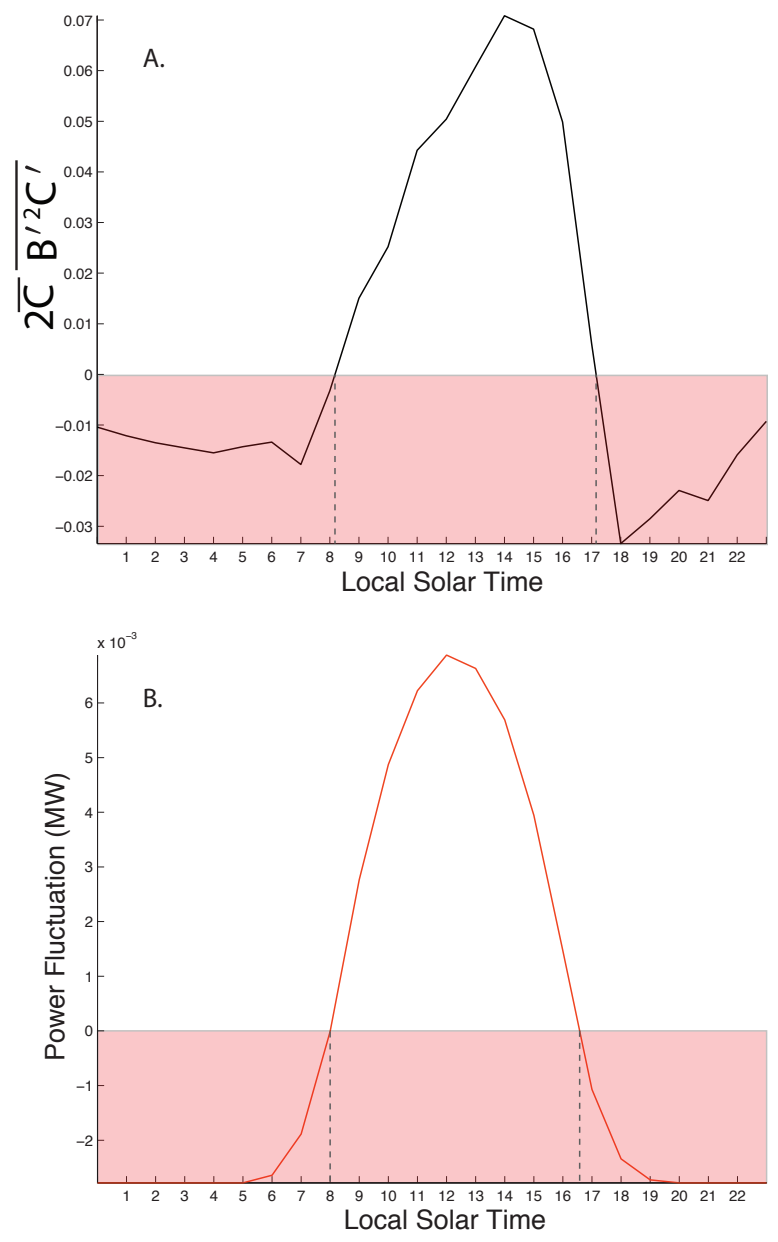

Figure 5: Plot of average diurnal behavior for the mean power times the covariance of the square of price fluctuations with the power fluctuations $\left(2 \bar{C} \overline{B^{\prime 2} C^{\prime}}\right)$ relative to relative to fluctuations from mean Price. 

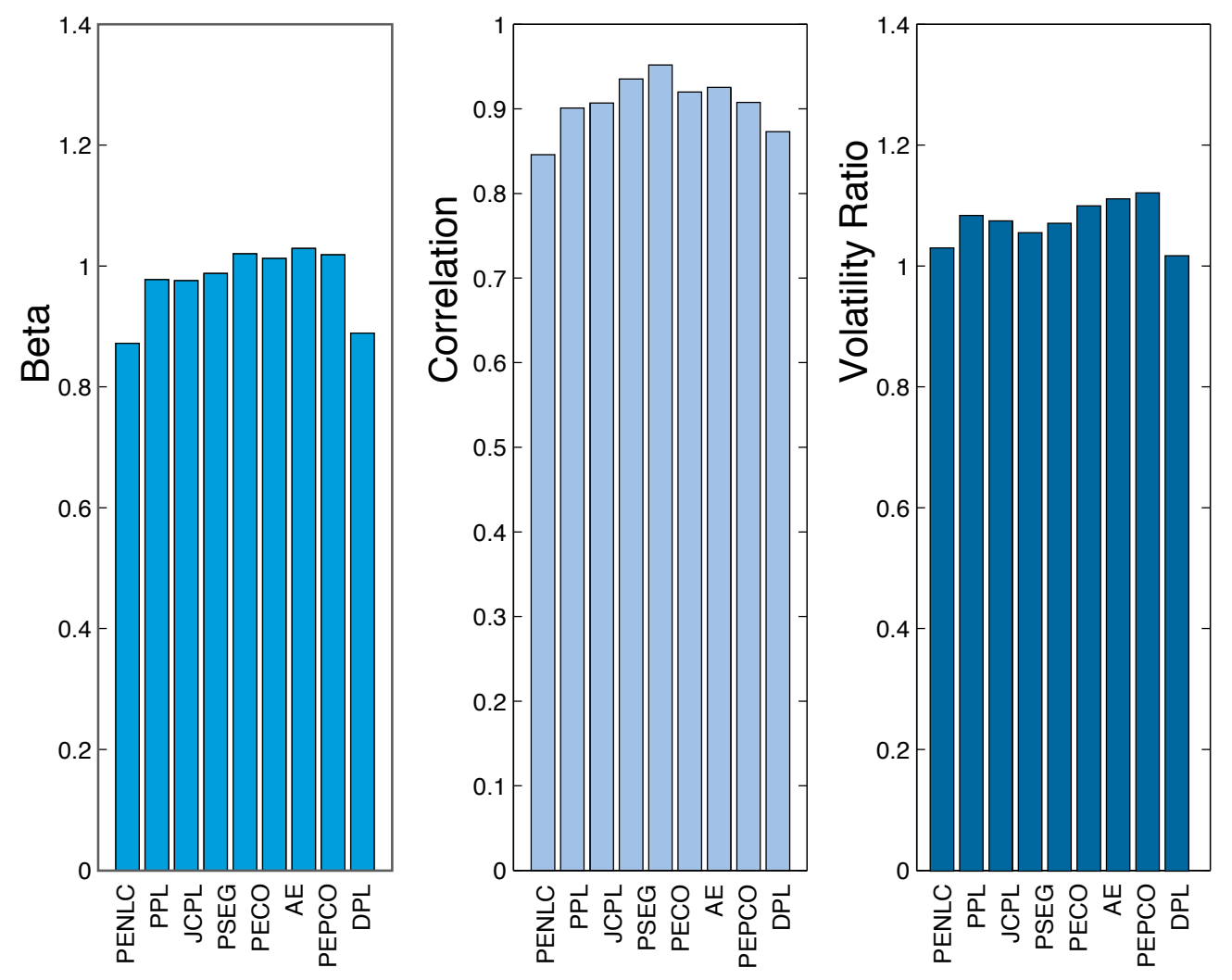

Figure 6: Annual values for beta, site correlation, and volatility ratio for revenue across all site. 


\section{Conclusion}

Revenue risk for a portfolio of photovoltaic arrays within a region has been described in terms of variability in the total revenue, accounting for seven years of data from PV power production and locational marginal pricing in the PJM region. Here, the dispersion of observed outcomes about expected values defines the risk for an individual site within a portfolio. The respective roles of price and power variability can be addressed by variance decomposition, so as to quantify the relative contributions of these input parameters to revenue risk, or total revenue variance. The beta metric for spatially distributed sites can be used to plan portfolios, so as to quantify and minimize revenue variability (i.e. hedge the revenue stream).

While total revenue variance is straightforward to calculate directly, the decomposition of terms for the revenue variance permits assessment of the roles of price and power, their diurnal and seasonal cycles, and the interplay of these cycles. Results in this paper demonstrate that revenue variance of a PV asset depends more on the diurnal, seasonal, and meteorological fluctuations than on the price behavior for a site. Furthermore, an increase in mean price will have a larger effect on revenue variance compared to a proportional increase in mean power production. The co-fluctuation of price and power terms account for the remaining third of the total revenue variance.

A solar beta can be defined appropriate to this class of problem that is analogous to the financial beta in portfolio analysis. Illustrating the solar beta for the PJM Mid-Atlantic region, it was observed that reductions in beta at the spatial perimeters of the study were due to change in climate regime (Great Lakes, subtropical coastal) and population-power consumption cycles. This suggests that structuring portfolios of PV assets across climate regimes may be a risk-reduction strategy for investors, assuming that costs and other site characteristics did not materially vary between sites. Furthermore, the correlation, and not the variation of revenue (volatility ratios), drives the beta behavior for sites.

The results of this study suggest that more benefit to the investor could be gained if solar energy production sites are considered in light of electricity market conditions and relationships with other sites rather than in isolation. Locations that have not traditionally been thought of as having the most advantageous conditions for peak power generation may have value as part of a geographically diverse portfolio. We have illustrated this effect in the U.S. Mid-Atlantic region but similar logic may hold in other locales (for example, 
280

examining the contribution of solar energy in areas such as northern Germany to a broader European portfolio).

\section{Acknowledgements}

The authors wish to thank Dr. Lucas Witmer for insight on data acquisition and processing, as well as initial TRNSYS modeling training. Blumsack acknowledges support from the National Science Foundation under grant CNS-133176. 
[1] USA Energy Information Administration, Electric power monthly, with data for december 2013, Tech. rep., US Department of Energy (February 2014).

URL http://www.eia.gov/electricity/monthly/pdf/epm.pdf

[2] J. C. Hull, Options, future and other derivatives, Pearson Education, Inc, 2009.

[3] J. C. Francis, D. Kim, Modern Portfolio Theory: Foundations, Analysis, and New Developments, John Wiley \& Sons, Hoboken, N.J, 2013.

[4] T. E. Hoff, R. Perez, Quantifying pv power output variability, Solar Energy 84 (2010) 1782-1793.

[5] R. Perez, K. Zweibel, T. E. Hoff, Solar power generation in the us: Too expensive, or a bargain?

URL http: //www . asrc. cestm.albany.edu/perez/2011/solval.pdf

[6] J. L. Bosch, J. Kleissl, Cloud motion vectors from a network of ground sensors in a solar power plant, Solar Energy 95 (2013) 13-20.

[7] M. Lave, J. Kleissl, Cloud speed impact on solar variability scalingApplication to the wavelet variability model, Solar Energy 91 (2013) $11-21$.

[8] V. Fung, J. L. Bosch, J. Kleissl, Cloud shadow speed sensor, Atmos. Meas. Tech. 7 (2014) 1693-1700.

[9] SolarAnywhere, Solar data (2013).

URL https://www. solaranywhere.com/Public/SelectData.aspx

[10] PJM, Pjm real-time energy markets (2013).

URL http://www.pjm.com/markets-and-operations/energy/realtime.aspx

[11] S. A. Klein, W. A. Beckman, J. W. Mitchell, J. A. Duffie, N. A. Duffie, T. L. Freeman, J. C. Mitchell, J. E. Braun, B. L. Evans, J. P. Kummer, R. E. Urban, A. Fiksel, J. W. Thornton, N. J. Blair, P. M. Williams, D. E. Bradley, T. P. McDowell, M. Kummert, D. A. Arias, TRNSYS 17: A transient system simulation program (2010). URL http://sel.me.wisc.edu/trnsys 
318

[12] SunPower Corporation, X-Series Solar Panels: Supplementary Technical Specifications, document no. 505915 rev a Edition (April 2013). URL http://us. sunpower.com/support/datasheets/

[13] H. A. Panofsky, J. A. Dutton, Atmospheric turbulence: models and methods for engineering aplications, John Wiley \& Sons, New York, 1984 .

[14] J. Rayl, G. S. Young, J. R. S. Brownson, Irradiance co-spectrum analysis: Tools for decision support and technological planning, Solar Energydoi:10.1016/j.solener.2013.02.029. 


\section{Appendix A. TRNSYS Component Specifications}

The following list specifies all of the inputs, outputs, and parameters of the various components of the TRNSYS simulation components used for this study. Information that is exchanged between the components is indicated by the arrowed links in Figure 2).

1. Data Reader

(a) Inputs

- GHI and DNI data accessed from outside source

- LMP data accessed from outside source

(b) Outputs

- GHI and DNI data to Irradiation Processor

- LMP data to Calculator and to Plot and Print

2. Irradiation Processor

(a) Inputs

- GHI and DNI from Data Reader

(b) Parameters

- Solar constant $(4899.6 \mathrm{~kJ} / \mathrm{h})$

- Ground reflectance (0.2 for all sites)

- Latitude (site specific - Table 1)

- Shift in solar time (site specific - Table A.4)

- Slope of surface (site specific - Table A.4)

(c) Outputs to $P V(w /$ Inverter $)$

- Total incident radiation on tilted surface

- Incidence angle on tilted surface

- Sky diffuse radiation on tiltled surface

- Beam radiation on tilted surface

- Array slope

3. 4-parameter $P V(w /$ Inverter $)$

(a) Inputs from Irradiation Processor

- Total incident radiation on tilted surface

- Incidence angle on tilted surface

- Sky diffuse radiation on tiltled surface 
- Beam radiation on tilted surface

- Array slope

(b) Parameters

- Load voltage (120 V)

- Module short-circuit current and open-circuit voltage (6.2 A, $21.6 \mathrm{~V})$

- Module maximum power current and voltage (5.84 A, 42.8 V)

- Number of cells (72)

- Number of modules in array (100)

- Module area $\left(1.244 \mathrm{~m}^{2}\right)$

- Ambient temperate (293 K)

- Semiconductor bandgap

(Monocrystalline - 1.12)

- Maximum inverter power (10000 W)

- Maximum inverter voltage $(1000 \mathrm{~V})$

(c) Outputs

- Maximum Power Point (W) to Calculator

- Maximum Power Point (W) to Plot and Print

4. Calculator

(a) Inputs

- LMP (\$/MWh) from Data Reader

- Maximum power (W) from $P V(w /$ Inverter $)$

(b) Calculations

- $\operatorname{LMP}(\$ / \mathrm{MWh}) \times \operatorname{Avg}$ Power $(\mathrm{W}) \times(1 \mathrm{MW} / 1 \mathrm{E} 6 \mathrm{~W})=$ Revenue $(\$ / h)$

(c) Outputs

- Revenue (\$) to Plot and Print

5. Plot and Print

(a) Inputs/Outputs

- LMP (\$/MWh) from Data Reader

- Maximum Power Point (W) from $P V(w /$ Inverter)

- Revenue $(\$ / \mathrm{h})$ from Calculator 
393 Note that two of the parameters for the Irradiation Processor are "site 394 specific" - the shift in solar time and the slope of the surface. Table A.4 gives 395 a list of these solar shifts and slope tilts for each location.

Table A.4: List of shift in solar hour and PV surface slope for all locations

\begin{tabular}{ccc}
\hline Site \# & Surface Slope & Solar Shift \\
\hline 1 & $34^{\circ}$ & -2.5 \\
2 & $34^{\circ}$ & -0.8 \\
3 & $33^{\circ}$ & +0.3 \\
4 & $33^{\circ}$ & +0.4 \\
5 & $32^{\circ}$ & -0.4 \\
6 & $32^{\circ}$ & +0.5 \\
7 & $31^{\circ}$ & -1.7 \\
8 & $30^{\circ}$ & -1.7 \\
9 & $29^{\circ}$ & -1.0 \\
\hline
\end{tabular}




\section{Appendix B. Variance Derivation Proof}

The following proof was developed to confirm the use of Eq. 3 for the variance analysis.

Let: $A=B * C$ and, by definition:

$$
\begin{gathered}
\operatorname{Var}(A)=\overline{(A-\bar{A})^{2}} \\
\operatorname{Var}(A)=\overline{(B * C-\overline{B * C})^{2}} \\
\operatorname{Var}(A)=\overline{B^{2} * C^{2}}-\overline{2 * B * C * \overline{B * C}}+\overline{\overline{B * C}^{2}} \\
\operatorname{Var}(A)=\overline{B^{2} * C^{2}}-2 * \overline{B * C} * \overline{B * C}+\overline{B * C}^{2} \\
\operatorname{Var}(A)=\overline{B^{2} * C^{2}}-2 * \overline{B * C^{2}+\bar{B}^{2}} \\
\operatorname{Var}(A)=\overline{B^{2} * C^{2}}-\overline{B * C}^{2}
\end{gathered}
$$

Recalling that a particular value of a variable $(X)$ equals the sum of that point's perturbation from the mean $\left(X^{\prime}\right)$ and the overall mean of the variable $(\bar{X})$. In other words: $B=\bar{B}+B^{\prime}$ and $C=\bar{C}+C^{\prime}$. Using this definition of the variance of $\mathrm{A}$ can be expressed in terms of the mean and fluctuating components of B and C. Note that by this definition the mean of any fluctuation is zero and mean quantities can be factored out of averages.

$$
\begin{aligned}
& \operatorname{Var}(A)=\overline{\left(\bar{B}+B^{\prime}\right)^{2}\left(\bar{C}+C^{\prime}\right)^{2}} \\
& -{\overline{\left(\bar{B}+B^{\prime}\right)\left(\bar{C}+C^{\prime}\right)}}^{2} \\
& \operatorname{Var}(A)=\overline{\left(\bar{B}^{2}+2 \bar{B} B^{\prime}+B^{\prime 2}\right)\left(\bar{C}^{2}+2 \bar{C} C^{\prime}+C^{\prime 2}\right)}
\end{aligned}
$$

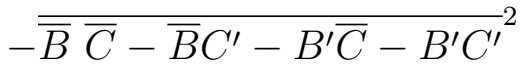

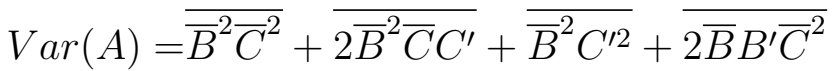

$$
\begin{aligned}
& +\overline{4 \bar{B} B^{\prime} \bar{C} C^{\prime}}+\overline{2 \bar{B} B^{\prime} C^{\prime 2}}+\overline{B^{\prime 2} \bar{C}^{2}} \\
& +\overline{2 B^{\prime 2} \bar{C} C^{\prime}}+\overline{B^{\prime 2} C^{\prime 2}}-\left(\bar{B} \bar{C}+\overline{B^{\prime} C^{\prime}}\right)^{2}
\end{aligned}
$$




$$
\begin{aligned}
\operatorname{Var}(A)= & \bar{B}^{2} \overline{C^{\prime 2}}+\bar{C}^{2} \overline{B^{\prime 2}}+2 \bar{B} \overline{B^{\prime} C^{\prime 2}}+2 \bar{C} \overline{B^{\prime 2} C^{\prime}} \\
& +2 \bar{B} \bar{C} \overline{B^{\prime} C^{\prime}}+\overline{B^{\prime 2} C^{\prime 2}}-{\overline{B^{\prime} C^{\prime}}}^{2}
\end{aligned}
$$

405 This equation was used to calculate the contributions of power (MW) and 406 price $(\$ / \mathrm{MWh})$ to revenue $(\$ / \mathrm{h})$, given that revenue is a product of hourly 407 average solar irradiation power and locational marginal pricing. 